\title{
Summary and Outlook
}

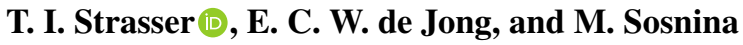

\section{Conclusions}

The expected large-scale roll out of Cyber-Physical Energy System (CPES) products and solutions during the next few years requires a multi-disciplinary understanding of several domains. The validation of such complex solutions gets more important as in the past and there is a clear shift from component-level to system-level testing. An integrated, cyber-physical systems-based, multi-domain approach for a holistic testing of smart grid solutions is currently still missing which is addressed by the ERIGrid approach [3].

Four main research priorities have been identified in this pan-European project to tackle the shortcomings in today's validation and testing of power systems and corresponding components. The research focus is put onto the development of a holistic validation methodology and the improvement of simulation-based methods, Hardware-in-the-Loop (HIL) approaches, and lab-based testing, which can be combined in a flexible manner. The integration and online connection of power systems/smart grid laboratories is also a challenging research and development task in ERIGrid. All these activities need to be supported by the training of researchers and power system professionals [3].

With the in the book described integrated pan-European Research Infrastructure (RI) approach in ERIGrid the following improved methods, services, and tools are being made available by the consortium members [3]:

\footnotetext{
T. I. Strasser ( $\square)$

AIT Austrian Institute of Technology, Vienna, Austria

e-mail: thomas.strasser@ait.ac.at

E. C. W. de Jong

KEMA B.V., Arnheim, The Netherlands
}

M. Sosnina

European Distributed Energy Resources Laboratories (DERlab) e.V., Kassel, Germany 
- Structured approach for defining a holistic description of validation and testing needs together with corresponding experiment descriptions,

- Improved co-simulation based approach with corresponding Functional Mock-up Interface (FMI)-based model libraries addressing the multi-domain and cyberphysical character of smart grid configurations,

- Improved HIL-based concepts analysing system-integration aspects of smart grid components,

- Possibility to couple co-simulation with lab experiments addressing systemintegration aspects,

- Evaluation of different Information and Communication Technology (ICT) and automation architectures, control concepts,

- Support for rapid-prototyping of components and analysing their behaviour in power systems,

- Support for evaluating different smart grid system configurations, and

- A set of educational material supporting the training of power systems professionals.

Summarizing, engineering and validation support will be critical for successful development of future CPES applications and solutions. Without the proper tool support many of the tasks will require immense manual efforts and will require engineers educated in multiple domains (energy system physics, ICT, automation and control, cyber-security, etc.). The available ERIGrid results provide a step forwards in the right direction, but more research and development efforts are still needed in the years to come as outlined below.

\section{Future Work}

In fact, current research show that already many aspects needed for a better engineering and validation of CPES are available. Nevertheless, many issues are still open and since the advancement of CPES technologies is still ongoing, new needs are constantly appearing. Especially also the integration of the electric energy system with other domains (thermal, gas, water/waste water, transportation, etc.) into a smart energy system [1] requires additional efforts. Therefore, the following list is an attempt to point out possible research and development directions that still needs to be explored from CPES validation point of view [2, 4]:

- Advanced RIs need to be developed which focus on the integration of different power and energy systems related areas (market issues, thermal topics, electric vehicle, etc.),

- A simplified access and corresponding services (facilitate future access by remote operation and coupling of both virtual and physical RI, etc.) to smart grid, smart energy systems, and renewable related RIs addressing challenging user needs is required, 
- Domain-specific adaptations of previously developed abstract validation procedures and corresponding concepts, methods, and tools are required to address advanced applications (low-inertia grids, microgrids, hybrid grids, etc.),

- Common and well understood reference scenarios, use cases, and test case profiles for smart energy systems need to be provided to power and energy systems engineers and researchers; also, proper validation benchmark criteria and key performance indicators as well as interoperability measures for validating smart grids and smart energy systems need to be developed, extended, and publically shared with domain professionals,

- A standardization of multi-domain CPES-based evaluation and testing procedures is necessary,

- Professionals, engineers, and researchers understanding smart grid and smart energy systems configurations in a multi-domain and cyber-physical manner addressing the upcoming energy transition need to be educated and trained on a broad scale.

The above listed open research and development issues are tackled by the successor project ERIGrid 2.0 ${ }^{1}$ which will be executed during the next years where several results are being provided open access to the domain of power and energy systems.

\section{References}

1. Mathiesen, B.V., Lund, H., Connolly, D., Wenzel, H., Østergaard, P.A., Möller, B., Nielsen, S., Ridjan, I., Karnøe, P., Sperling, K., et al.: Smart energy systems for coherent $100 \%$ renewable energy and transport solutions. Appl. Energy 145, 139-154 (2015)

2. Neis, P., Wehrmeister, M.A., Mendes, M.F.: Model driven software engineering of power systems applications: literature review and trends. IEEE Access 7, 177761-177773 (2019)

3. Strasser, T., Pröstl Andrén, F., Widl, E., Lauss, G., et al.: An integrated pan-European research infrastructure for validating smart grid systems. e \& i Elektrotechnik und Informationstechnik 135(8), 616-622 (2018)

4. Strasser, T.I., Andrén Pröstl, F.: Engineering and validating cyber-physical energy systems: needs, status quo, and research trends. In: International Conference on Industrial Applications of Holonic and Multi-Agent Systems, pp. 13-26. Springer (2019)

\footnotetext{
${ }^{1}$ https://erigrid2.eu.
} 
Open Access This chapter is licensed under the terms of the Creative Commons Attribution 4.0 International License (http://creativecommons.org/licenses/by/4.0/), which permits use, sharing, adaptation, distribution and reproduction in any medium or format, as long as you give appropriate credit to the original author(s) and the source, provide a link to the Creative Commons license and indicate if changes were made.

The images or other third party material in this chapter are included in the chapter's Creative Commons license, unless indicated otherwise in a credit line to the material. If material is not included in the chapter's Creative Commons license and your intended use is not permitted by statutory regulation or exceeds the permitted use, you will need to obtain permission directly from the copyright holder. 